\section{Pneumocystis pneumonia and disseminated toxoplasmosis in a male homosexual}

Within the past two years 788 cases of the apparently new and potentially lethal syndrome of acquired immune deficiency have been reported in the United States. ${ }^{1}$ Only three cases have been reported in the United Kingdom. ${ }^{2-4}$ We report a case in a previously healthy 37 year old male homosexual who was initially diagnosed and treated for pneumocystis pneumonia and subsequently died of widespread toxoplasmosis.

\section{Case report}

The patient presented with an eight week history of malaise, non-productive cough, night sweats, diarrhoea, anorexia, and weight loss. He had previously acquired syphilis, hepatitis B, and on four occasions gonorrhoea. His recreational drugs were amyl nitrite and lysergide. His male partner was apparently well.

Pneumocystis pneumonia was suspected in view of his history, and bilateral patchy shadowing was seen on chest $x$ ray films. He had no lymphadenopathy. Initial investigations showed haemoglobin $9.5 \mathrm{~g} / \mathrm{dl}$ and white cell count $4.9 \times 10^{9} / 1$ (neutrophils $89 \%$, lymphocytes $4 \%$, monocytes $5 \%$ eosinophils $2 \%$ ). Lactate dehydrogenase activity was greater than $800 \mathrm{IU} / \mathrm{l}$. Serum albumin concentration was $27 \mathrm{~g} / 1$ and serum immunoglobulins were in the normal range except for a slightly raised concentration of IgG to $14 \cdot 6 \mathrm{~g} / \mathrm{l}$. Transbronchial biopsy failed to show Pneumocystis carinii. Treatment was started with intravenous co-trimoxazole $(240 \mathrm{mg}$ trimethoprim and $1200 \mathrm{mg}$ sulphamethoxazole twice daily). The pneumocystis immunofluorescent antibody titre rose from $1 / 16$ on admission to $1 / 128$ three weeks later. After 48 hours the co-trimoxazole regimen was altered to $160 \mathrm{mg}$ trimethoprim and $800 \mathrm{mg}$ sulphamethoxazole by mouth six times a day. There was no serological evidence of infection with cytomegalovirus. No virus was isolated from stool, cerebrospinal fluid, blood, urine, or throat. The toxoplasma dye test titre was $1 / 128$. Apart from detecting a negative Mantoux response, we were unable to study delayed hypersensitivity reactions.

After 13 days' treatment, despite some clinical improvement, his temperature remained raised and intravenous co-trimoxazole was given for a further 15 days $(400 \mathrm{mg}$ trimethoprim and $2000 \mathrm{mg}$ sulphamethoxazole twice a day). Serum concentrations of trimethoprim were above $5 \mathrm{mg} / \mathrm{l}$. Three weeks later his symptoms recurred and chest $x$ ray films again showed abnormalities, so pentamidine isethionate was given (in a dose of $200 \mathrm{mg}$ intramuscularly once a day increasing to $250 \mathrm{mg}$ after two days). After six days' treatment severe pyrexia developed $\left(40 \cdot 5^{\circ} \mathrm{C}\right)$ and he became delirious, hypotensive (blood pressure $80 / 50 \mathrm{~mm} \mathrm{Hg}$ ), and hypovolaemic. The drug was stopped because of possible toxicity, and despite correction of the fluid deficit he died suddenly the next day.

Necropsy was performed two days after death, and histological examination of the brain, pituitary, myocardium, liver, lungs, and trachea showed large numbers of tissue cysts of Toxoplasma gondii with myocarditis and encephalitis. There was no evidence of Pneumocystis carinii, Kaposi's sarcoma, or lymphoma. The toxoplasma serology on stored sera was then reviewed. On admission the toxoplasma dye test titre had been 1/128; six weeks later it had not changed appreciably $(1 / 256)$, but a week before death the titre had risen to $1 / 2056$. Toxoplasma specific IgM could not be detected in any sample.

\section{Comment}

The diagnosis of pneumocystis pneumonia in our patient was based on a rising antibody titre. Transbronchial biopsy techniques are less reliable than open lung biopsy, and this may have accounted for our failure to detect the organism.

There was unequivocal histological evidence of disseminated toxoplasmosis at necropsy despite the lack of toxoplasma specific IgM in the serum. While conventional treatment for toxoplasmosis is with pyrimethamine and a sulphonamide, co-trimoxazole is an alternative agent. It is thus possible that the treatment directed at the pneumocystis pneumonia may have diminished the immune response to toxoplasmosis by coincidentally treating the latter, albeit ineffectively.

The diagnosis of opportunistic infections in patients with the acquired immune deficiency syndrome is complex: infections may be multiple, invasive techniques may be required, and serological or histological evidence of infection may be absent. In the management of a patient with the syndrome the doctor must be constantly aware of the possibility of further infections developing. The high mortality rate of the syndrome $(>60 \%$ for cases diagnosed before June 1981) surely justifies the use of invasive techniques for diagnosis.

We thank Professor W I Cranston for permission to report his patient Dr I E West and Dr H Pambakian, who reported the histology, for helpful discussions, and Dr B Jameson and Dr D G Fleck for their valuable assistance with the pneumocystis and toxoplasma serology.

1 Ammann A, Cowan M, Wara D, et al. Possible transfusion-associated acquired immune deficiency syndrome (AIDS)-California. Morbidity and Mortality Weekly Report 1982;31:652-4.

2 Du Bois RM, Branthwaite MA, Mikhail JR, et al. Primary pneumocystis carinii and cytomegalovirus infections. Lancet $1981 ;$ ii:1339.

3 Oswald GA, Theodossi A, Gazzard BG, et al. Attempted immune stimulation in the "gay compromise syndrome." $\mathrm{Br} M e d \mathcal{f} 1982 ; 285: 1082$.

4 Maurice PDL, Smith NP, Pinching AJ. Kaposi's sarcoma with benign course in a homosexual. Lancet $1982 ; \mathrm{i}: 571$.

5 Task force on acquired immune deficiency syndrome, Centers for Disease Control. Update on acquired immune deficiency syndrome (AIDS)United States. Morbidity and Mortality Weekly Report 1982;31:507-14.

(Accepted 4 March 1983)

Departments of Microbiology and Medicine, St Thomas's Hospital, London SE1 7EH

W R GRANSDEN, MA, MB, lecturer

$P$ M BROW iN, MB, MRCP, lecturer

Correspondence to: Dr W R Gransden.

\section{Finger sepsis: possible complication of self monitoring of blood glucose concentrations}

Self monitoring of blood glucose concentrations has greatly facilitated the achievement of improved glycaemic control in diabetics. ${ }^{1}$ Capillary blood samples are usually obtained by pricking a finger with a small stylet, and except for some coarsening of the $\operatorname{skin}^{1}$ the procedure has been remarkably free of complications. ${ }^{2}$ We report two cases in which a more serious complication occurred.

\section{Case reports}

Case 1-A 42 year old man with diabetes of 20 years' duration and multisystemic complications began monitoring his blood glucose concentrations in 1980. Three months later a bovine graft was inserted in his left arm for haemodialysis. A year later an abscess developed over the tip of the left index finger. Despite antibiotic and surgical treatment of the abscess on two occasions the area of inflammation enlarged and an $x$ ray film (figure) showed severe necrosis and osteomyelitis of the distal phalanx, necessitating amputation at the distal interphalangeal joint.

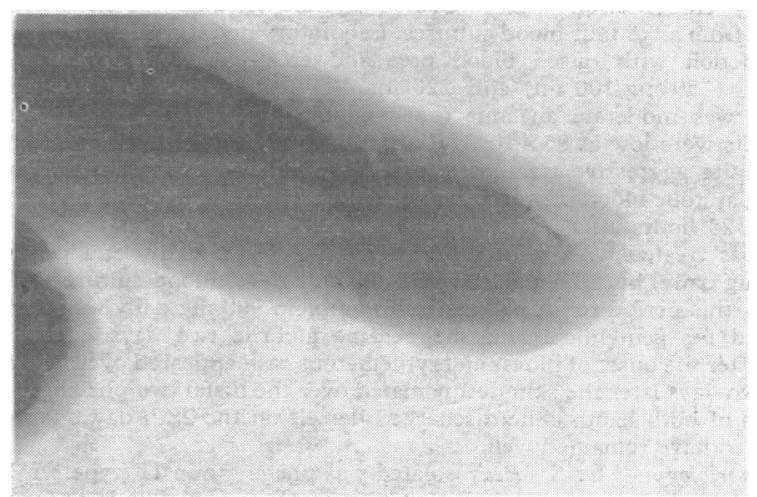

Radiograph of finger, showing severe necrosis and osteomyelitis of distal phalanx.

Case 2-A 40 year old woman with diabetes of 38 years' duration and extensive complications began monitoring her blood glucose concentrations after renal transplantation in 1979. She had shunts in both forearms, which had been created for vascular access for haemodialysis in the preceding year. A year later sepsis developed in the tip of her left middle finger, which did not improve with local treatment. Six months later the tips of the right index and middle fingers became ulcerated. Although she stopped obtaining samples from these three fingers, the infections did not clear and by early 1982 gangrene had developed in the digits, necessitating distal amputation of all affected tissue. 


\section{Comment}

Self monitoring of blood glucose concentrations has had a major impact on the management of diabetes and when used with either intensified conventional treatment or continuous subcutaneous insulin infusion allows euglycaemia to be achieved. ${ }^{3}$ The technique has been notably free of problems except for one reported case of dextrose contamination of the finger tips. ${ }^{4}$

Healthy skin will tolerate repeated puncture without ill effects. In diabetes, however, several adverse factors may be compounded and lead to tissue necrosis. Impaired blood supply, neuropathy, abnorma synthesis of collagen, and the fact that more intensive monitoring is required as the disease progresses lead to an increased risk of infection initially followed by impaired healing, which impedes recovery. As in our patients, the creation of shunts in the forearm for vascular access in haemodialysis may further compromise blood flow to the fingers. Impaired peripheral sensation, often present in these patients, may aggravate matters since the patient may continue to use an obviously damaged but pain free site. The increased risk of infection during immunosuppressive treatment after renal transplantation may be another compounding factor.

In case 1 problems occurred in only one finger, which may represent an unfortunate lapse of aseptic technique; the increased risk of infection in diabetes, however, was shown by the poor outcome despite prompt antibiotic and surgical treatment. The ease of prevention compared with the difficulty of eradicating established infection must be emphasised to diabetics using this self monitoring technique. Our second patient had more severe vascular disease, neuropathy, and visual impairment that led to her sometimes having to use several sticks for a single capillary blood sample. She also took at least four and often seven readings a day. These factors all contributed to the occurrence of sepsis on several fingers and emphasise the increased likelihood of problems when a partially sighted patient obtains capillary blood samples from fingers with decreased sensation. Advice should also be given to diabetics with haemodialysis shunts to avoid getting samples from the fingers of the arm containing the shunt and on the appropriateness of using the side of the finger rather than the finger pulp.

Although we use self monitoring in many of our diabetics and all of our diabetics with renal failure, who usually have associated neuropathy, this complication is rare. Nevertheless, it is important to prevent these lesions and to give careful attention to ulcers when they appear.

' Skyler JS, Lasky IA, Skyler DL, Robertson EG, Mintz DH. Home blood glucose monitoring as an aid in diabetes management. Diabetes Care $1978 ; 1: 150-7$.

2 Walford S, Gale EAM, Allison SP, Tattersall RB. Self monitoring of blood glucose. Lancet $1978 ; 1: 732-5$.

${ }^{3}$ Reeves ML, Seigler DE, Ryan EA, Skyler JS. Glycemia control in insulindependent diabetes mellitus: comparison of outpatient intensified conventional therapy with continuous subcutaneous insulin infusion. Am f Med 1982;72:673-80.

${ }^{4}$ Kinmonth AL. Home blood glucose monitoring: a sticky artefact. $\mathrm{Br}$ Med f $1981 ; 282: 272-3$.

(Accepted 22 February 1983)

Diabetes/Endocrinology Unit and Renal Transplant Division,

University of Miami, Miami, Florida, USA

EDMOND A RYAN, MRCPI, instructor in medicine

JOSHUA MILLER, MD, chief of transplantation division

JAY S SKYLER, MD, associate professor of medicine and paediatrics

Correspondence to: Dr E A Ryan.

\section{Combination treatment with tamoxifen and aminoglutethimide in advanced breast cancer}

Tamoxifen and aminoglutethimide are effective drugs in the endocrine treatment of advanced breast cancer with response rates of around $30 \%{ }^{12}$ They act by different mechanisms, ${ }^{12}$ and by analogy with combination chemotherapy we studied their efficacy in combination in the treatment of advanced breast cancer. We compared our results with those obtained in a previous trial comparing the two drugs as single agents."

\section{Patients, methods, and results}

Sixty two consecutive patients with histologically proved advanced breast cancer were treated with tamoxifen $10 \mathrm{mg}$ by mouth twice daily in combination with aminoglutethimide $250 \mathrm{mg}$ by mouth three times daily (increasing to four times daily after two weeks if toxicity permitted) with hydrocortisone $20 \mathrm{mg}$ twice daily. Standard criteria for staging disease and for defining objective response were used as previously described. ${ }^{2}$ Median age was 56 years (range 31-77 years); six patients were premenopausal, eight perimenopausal, and 48 postmenopausal or postoophorectomy.

Twenty three of the 62 patients $(37 \%)$ achieved an objective response and seven showed stable disease for at least three months. Responses were seen for all sites of disease, including soft tissue (12/25 patients), bone (8/29), lung $(5 / 16)$, and liver $(2 / 6)$. The figure shows the duration of the response calculated by life table analysis compared with that for tamoxifen and for aminoglutethimide used as single agents. ${ }^{2}$ Median duration of response was

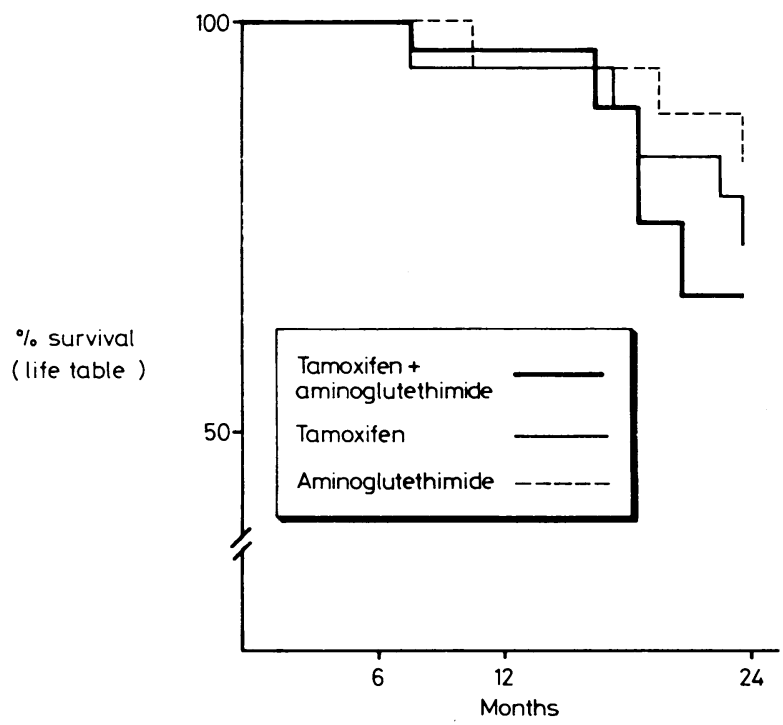

Duration of response from start of treatment (life table analysis) obtained with combined tamoxifen and aminoglutethimide compared with responses obtained with tamoxifen alone and aminoglutethimide alone. ${ }^{2}$

16 months, which is not significantly different from the duration of response obtained with each agent alone. Median survival of patients who responded has not yet been reached, but at 24 months after the start of treatment the predicted survival rate for combination treatment was $67 \%$, compared with $75 \%$ for tamoxifen alone and $82 \%$ for aminoglutethimide alone. Side effects were similar to those previously described for aminoglutethimide alone ${ }^{2}$ and included initial lethargy or drowsiness $(35 \%)$, a self limiting rash $(27 \%)$, nausea $(11 \%)$, and depression $(18 \%)$. Four patients $(6 \%)$ could not tolerate treatment.

\section{Comment}

This study suggests that a combination of tamoxifen and aminoglutethimide has no advantage over either agent used alone in terms of the rate or duration of response. Indeed, our results reflect the consistent reporting of similar rates and durations of response obtained with all major forms of endocrine treatment used alone in advanced breast cancer. ${ }^{3}$

In the absence of therapeutic benefit combined endocrine treatment has disadvantages. It is more expensive and likely to be associated with increased toxicity. More importantly, the opportunity offered by sequential treatment for a second response after relapse with first line treatment is lost. This might eventually be reflected in a shortened overall survival with combination endocrine treatment, although such a disadvantage would take time to emerge and has not so far been seen.

Different combinations of endocrine treatment might possibly be more effective than this one, although reports so far are not encouraging. Two Danish trials failed to show any benefit for tamoxifen combined with medroxyprogesterone acetate or diethylstilboestrol over tamoxifen alone. ${ }^{4}$ Preliminary data from a triple combination trial with tamoxifen, aminoglutethimide, and danazol show a higher response rate but no difference in duration of response or survival compared with tamoxifen alone, ${ }^{5}$ and a trial of combined aminoglutethimide and danazol showed a significantly inferior rate of 